\title{
Agonistic Anti-OX40 Monoclonal Antibody INCAGN01949
}

National Cancer Institute

\section{Source}

National Cancer Institute. Agonistic Anti-OX40 Monoclonal Antibody INCAGN01949. NCI

Thesaurus. Code C129967.

An agonistic human immunog lobulin G1 (IgG1) monoclonal antibody that recognizes the co-stimulatory receptor OX40 (CD134; TNFRSF4), with potential immunostimulatory and antineoplastic activities. Upon administration, agonistic anti-OX40 monoclonal antibody INCAGN01949 selectively binds to and activates OX40 on activated T-cells, thereby potentiating T-cell receptor (TCR) signaling. OX40 activation inhibits regulatory T-cell (T reg)-mediated suppression of effector T-cells, induces the proliferation of memory and effector T-lymphocytes and modulates cytokine production. In the presence of tumor-associated antigens (TAAs), this may promote an immune response against the TAA-expressing tumor cells. In addition, the IgG1 Fc region of INCAGN01949 binds to and co-eng ages with the IgG Fc-gamma receptor III (FcgammaRIII; CD16) expressed by immune effector cells; thus, binding activates FcgammaRIII-mediated signaling and facilitates the selective depletion of intratumoral Tregs, thereby further enhancing the cytotoxic T-lymphocyte (CTL)-mediated tumor cell response. OX40, a cell surface glycoprotein and member of the tumor necrosis factor (TNF) receptor superfamily, is expressed on $\mathrm{T}$-lymphocytes and provides a co-stimulatory signal for the proliferation and survival of activated T-cells; OX40 stimulation abrogates the immunosuppressive tumor microenvironment. 